\title{
Generation of elastic geodesic gridshells with anisotropic cross sections
}

\author{
Charles Haskell $^{1}$, Nicolas Montagne ${ }^{1,2}$, Cyril Douthe $^{1}$, Olivier Baverel ${ }^{1,3}$, Corentin Fivet $^{2}$
}

\begin{abstract}
Geodesic gridshells are shell structures made of continuous elements following geodesic lines. Their properties ease the use of beams with anisotropic cross-sections by avoiding bending about their strong axis. However, such bending may arise when flattening arbitrary geodesic grids, which forbids their initial assembly on the ground. This study provides a process to design elastic geodesic gridshells, i.e. gridshells that minimise bending moments in both formed and nearflat configurations. The generation process first brings a target geodesic network onto a plane by maintaining arc lengths. The flat mesh is then relaxed to minimise its main curvatures and hence bending moments in its members. The result is an elastic geodesic gridshell that can be assembled flat on the ground and then lifted up into its target surface. The method is applied to the design of six geodesic gridshells made of reclaimed skis.
\end{abstract}

\section{Keywords}

gridshell, geodesic lines, parametric modelling, skis, reuse, construction-aware form-finding

\section{Introduction}

This study falls within the research domain dedicated to the generation of expressive, irregular shell structures whose construction process is eased by tailored global geometric features. In particular, the useful properties of elastic gridshells are here combined with those of geodesic shells, as a means to enable the rapid construction of shells made of elements with anisotropic rectangular cross-sections, such as for instance, reclaimed skis.

Elastic gridshells are appealing for both architects and engineers for their capacity to combine lightness, aesthetics and inherent shape stability. These structures are obtained by elastic deformation of an initially flat bidirectional regular grid of articulated bars. The deformed grid is then braced to lock the geometry and ensure structural stiffness. Such systems were developed by Frei Otto in the 1970's ${ }^{1}$, and have been used in several projects since the 2000s (gridshell in timber laths ${ }^{2}$; gridshell in composite materials ${ }^{3}$; gridshell in reclaimed skis ${ }^{4}$ ). The flat construction and simple erection process of elastic gridshells make them very attractive compared to other systems. Recently Bouaya et al. ${ }^{5}$ addressed the problem of optimising the position of the regular grid on a target surface through genetic algorithm with the aim to reduce internal stresses and thus material consumption. Alternatively, Maia-Avelino et al. ${ }^{6}$ studied patches of regular grids to widen the solution space of elastic gridshells. Furthermore, Douthe et al. ${ }^{7}$ identified a special family of surfaces suitable for the construction of elastic gridshells covered with flat quadrangular panels.

Geodesic shells have been introduced by Pirazzi and Weinand ${ }^{8}$ and are grid structures whose members follow geodesic lines on a target surface. This gridshell type is buildable from initially-straight rectangular beams. In fact, an element following a geodesic line is not bent around the normal vector of the surface. Thus, using a rectangular cross-section beam tangent to the surface, such a path avoids bending around the strong axis of the member. Although the typology of a geodesic gridshell can be optimised, its construction remains a challenge. The members of the structure are positioned and assembled one after the other, requiring to work at height and resulting in a long and tedious construction process on site. Inspired by the erection process of elastic gridshells, Soriano et al. ${ }^{9}$ studied the construction of geodesic shells from an initially flat grid of laths. The empirical exploration led to the identification of several specific layouts. However, as shown by Lagally ${ }^{10}$, a mesh cannot be flattened in general. Pillwein et al. ${ }^{11}$ also explored the planar-to-spatial deployment of a geodesic gridshell. In their study, the continuous geodesic lines are only connected at their ends, and the inner pinned connections are not fixed on the ground. Thus, off-ground work is required to fix them.

Inspired by Soriano et al. ${ }^{9}$, the objective of this paper is to develop a strategy for the projection of geodesic gridshells on the ground, in order to facilitate pre-fabrication, transportation and erection of such structures. In section 1, two different methods are presented. The first one starts with a network of intersecting curves and rebuilds it using an adaptation of the "Compass method" introduced by Otto et al. ${ }^{12}$. The second methods takes a quadrangular mesh made of a bidirectional grid of geodesic curves. Then, for both methods, a mesh relaxation algorithm is used to find a planar grid with minimal bending energy and to allow the introduction of assembly clearance in the model.

\footnotetext{
${ }^{1}$ Laboratoire Navier, UMR 8205, Ecole des Ponts ParisTech, France

${ }^{2}$ Structural Xploration Lab, ENAC, EPFL, Switzerland

${ }^{3}$ GSA ENS Architecture Grenoble, France
}

Email: charles.haskell@eleves.enpc.fr 
The methods are applied to the design of three elastic geodesic gridshells made of reclaimed skis, inspired by Colabella et al. ${ }^{4}$ and presented on Figure 1. Reusing skis addresses both an economic and ecological issue. Hundreds of thousands of skis are thrown away each year in France and in Europe. Made of composite materials, skis are generally not recycled and directly burnt or moved to landfills. Instead, reusing discarded skis in new applications increases their service life and avoids the manufacturing of other materials. The quality of such circular strategy depends on how much of the embedded technology in the skis is well utilised in the new application. In other words, the quality of the reclaim depends on whether the new application implies a premature down-cycling of the component or not. From a mechanical point of view, skis are high-performance beams with a rectangular cross-section. It is therefore wise to arrange skis along a geodesic network, with their strong bending axis oriented normal to the target surface so that they can take high curvatures with minimum strain energy in one direction, while maintaining high stiffness in the other directions. Still, the flattening of a geodesic network introduces bending moments along strong axes and critical curvatures must be checked accordingly.

Section 2 describes the overall methodology. The geometric generation algorithms are then applied to three different gridshells in section 3. Section 4 studies the mechanical behaviour of these gridshells if constructed with reclaimed skis. Section 5 presents corresponding physical models. A discussion bridging results from all previous sections is given in section 6 .

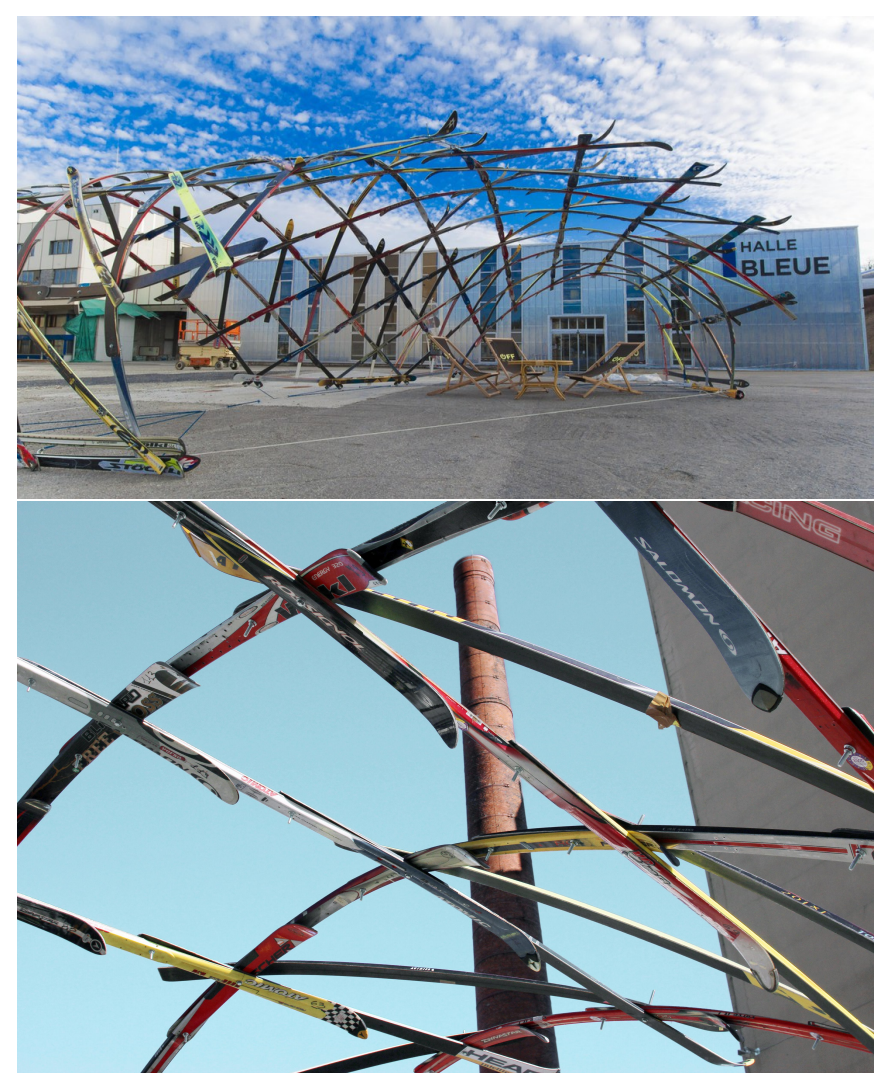

Figure 1. Ski pavilion at the EPFL, Lausanne, Switzerland

\section{Contents}

\section{Introduction}

Methodology

2

Generation of geodesic curves . . . . . . . . 2

Grid flattening . . . . . . . . . . . 3

Minimum bending energy curvature relaxation . . . 3

Mechanical validation . . . . . . . . . . . 4

\section{Geometric models}

Case I (tunnel) . . . . . . . . . . . . . . . . . . . . . . . . 4

Case II (dome) . . . . . . . . . . . . . . . . . . . 4

Case III (wave) . . . . . . . . . . . . . 6

Case IV (sheet) . . . . . . . . . . . . 6

Case V (rhombus) . . . . . . . . . . . . . . . . 6

Case VI (toroid) . . . . . . . . . . . . . . . . . . 6

Recap ............... 6

\section{Mechanical models}

Critical curvatures of bent skis and assembly prestress .................. 7 Material properties . . . . . . . . . . 7

Elastic curvature . . . . . . . . . 7

Critical curvature . . . . . . . . . . 7

Assembly prestress . . . . . . . . . . . . 7

Number of layers, bracing, and stiffness . . . . . 8

Model assumptions . . . . . . . . . . . 8

case I (tunnel) . . . . . . . . . . . . . 8

case II (dome) . . . . . . . . . . . . . . . 8

case III (wave) . . . . . . . . . . . . 9

\section{Physical model}

\section{Discussion}

Comparison of the parametric model and mechan-

ical calculation results . . . . . . . . . 10

Limits of the flattening methods . . . . . . . . 10

Limits of the numerical simulations . . . . . . 10

Limits of the physical models . . . . . . . . . . 10

\section{Conclusion}

\section{Methodology}

The following four subsections detail the main steps of the generation process, as synthesised on figure 2. First, a network of geodesic curves is obtained from a target surface. From there, the network is flattened, its mesh is relaxed, and the mechanical behaviour of the new gridshell is eventually validated.

\section{Generation of geodesic curves}

The design of geodesic gridshells requires an initial surface defining the volume to enclose. From this initial geometry, geodesic curves are drawn one by one on the surface. The drawing process starts either with each curve extremities, or with a start point and the curve's tangent at that point. In the first case, the drawing method reflects the definition of geodesic lines as the shortest path between two points. In the second case, geodesic lines are understood as curves with vanishing geodesic curvature. In the following, the first 


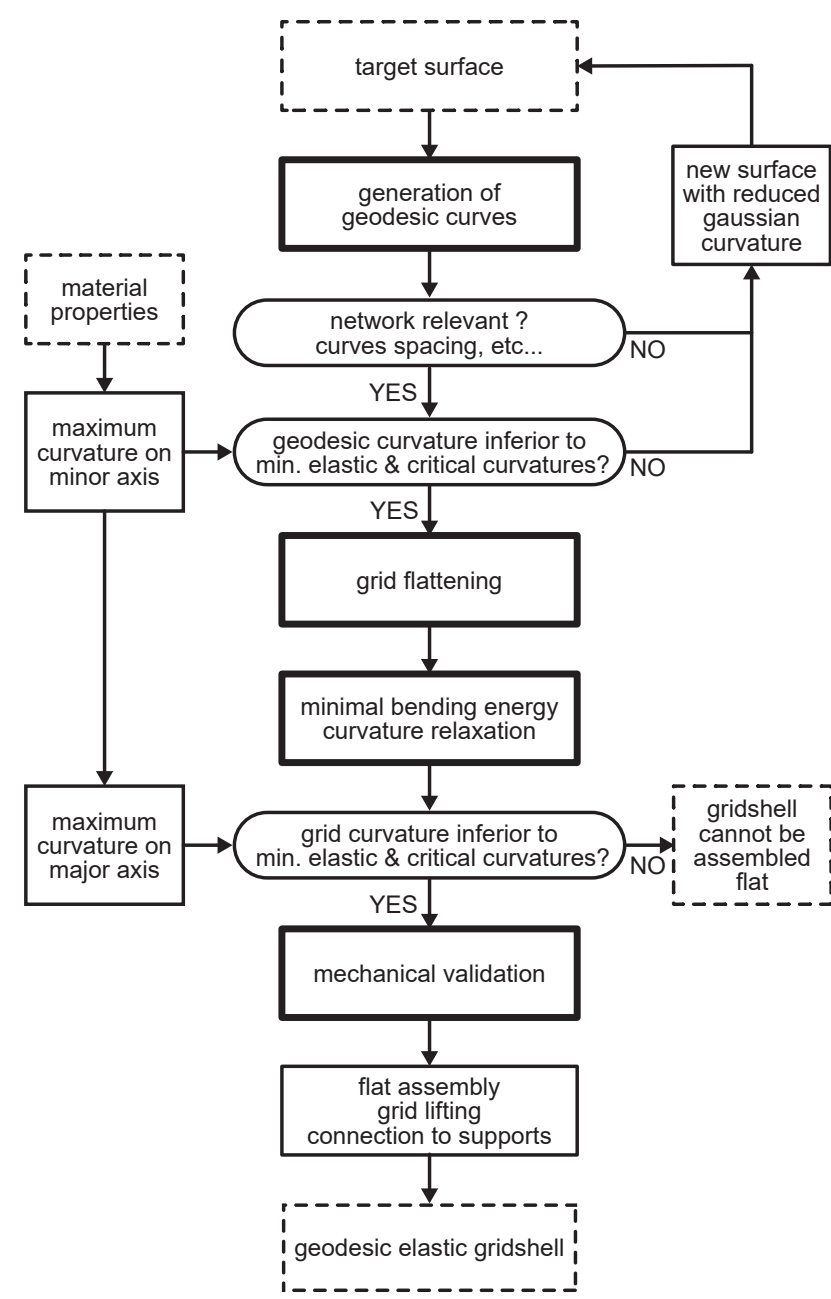

Figure 2. Design workflow: dashed boxes are inputs or outputs; thick boxes correspond to processes explained in methodology sub-sections

drawing method is preferred as it allows to rigorously control the position of the ground supports. The generated geodesic lines form a network of curves that smoothly approximates the target shape.

\section{Grid flattening}

Two different methods are here presented to bring the initial network of geodesic curves into a flat configuration. Both make use of the parametric modelling software Grasshopper.

The first method, method " $A$ ", is based on the Compass method, as first introduced by Frei Otto et al. ${ }^{12}$ for the design of elastic gridshells, and more particularly for the generation of regular grids with fixed edge length, called Chebyshev nets, on any given surface. However, the process here is adapted to map grids with varying edge lengths. The process, Figure 3 is applicable to any quadrangular grid, including irregular ones. For a given geodesic gridshell, the arc lengths between the intersections of one direction of geodesic lines and the other direction of curves in the grid network are assessed. The intersection between two curves is then transferred onto the plane by retrieving the crossing of two arc-circles, centred at the starting point of the curves and whose radii are the measured arc lengths. This process is repeated for each curve intersection, called node, in the network. Finally, by connecting these nodes, planar curves are obtained, corresponding to the flattened curve network.

Alternatively, a second method, method "B", to flatten the network of geodesic curves employs soft constraints on arc lengths but combines them with soft constraints on the quadrilaterals of the associated mesh. From the geodesic curves approximating the target surface, a network of intersecting discrete curves is created. Each initial geodesic is segmented into a polyline whose vertices are located at the crossing with the other curves. Then, a mesh is generated from the two-way grid of polylines. The vertices and segments of the polylines become respectively the vertices and edges of the mesh, and the quadrilaterals formed by the curve network become the faces. The mesh is eventually flattened using an optimisation procedure that contains soft constraints to maintain the lengths of the edges of each quadrilaterals ${ }^{13,14}$. Additional constraints can be set on angles between consecutive segments of each polyline. By setting a null angle target, the polylines are flattened with a minimum curvature. This provides an initialising step before relaxing the flat grid.

The two flattening methods differ in whether initial segment arc-lengths are maintained or not. The first method maintains all arc-lengths by definition. Because the discretization of the mesh in the second method is dictated by the intersection between the geodesic curves, edge lengths in the mesh may not accurately represent the arc lengths of the geodesic curves, especially if the curvature is high. However, the second method takes into consideration the inplane bending of the flattened grid, and thus provides with better initialisation for the next step, i.e. mesh relaxation.

\section{Minimum bending energy curvature relaxation}

In the previous section, two methods to flatten geodesic grids have been presented. In both cases, the resulting grid does not account for material properties of beam members, neither their cross section, nor their material stiffness. Thus, the flattened grid may not be buildable with anisotropic beams because the assembly might cause bending along strong axes, and important forces would have to be exerted to bend them, leading to buckling or even breaking of members.

Therefore, mesh relaxation is used to reduce beam deformations that are intrinsic to the flattening operation. The objective that is minimised is the bending energy of all continuous lines defining the flattened grid. To this end, the polylines obtained in the previous flattening process are modelled as Rods. This component has two key components for the flattening process: length factor and angle factor. The length factor is set to 1 , meaning that the beams length must remain constant during the flattening. The angle factor is set to 0 , meaning that the target angle between two neighbouring lines is null, this would force the flattened polylines to be in a configuration where they are as straight as possible, thus minimising the major axis bending moment. The On-plane component is used to maintain the nodes on the same plane and avoid out-of-plane deformations. All these parameters are then gathered in the dynamic relaxation solver Kangaroo ${ }^{13}$. The algorithm converged in seconds for all case studies presented in this paper.

Assembly clearance is introduced in the geometric model to simulate the sliding behaviour of connections due to loose 


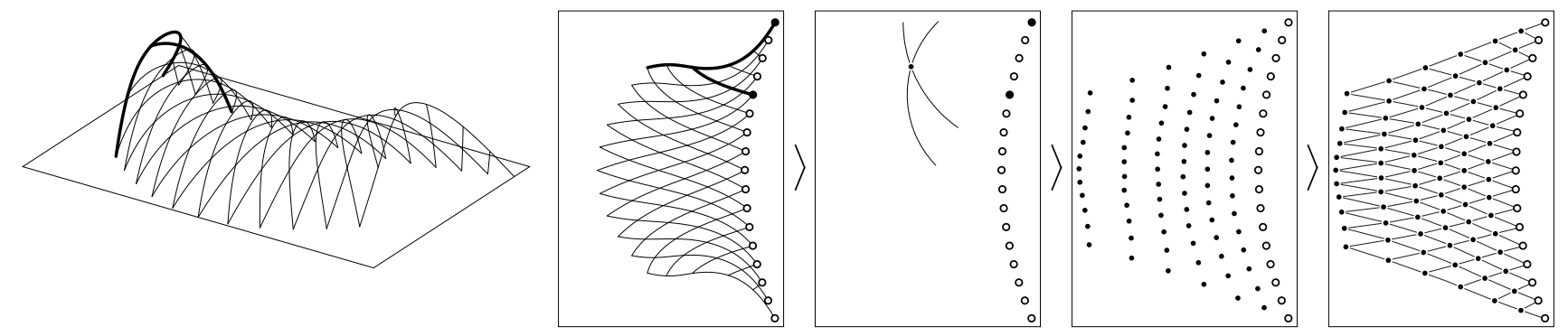

Figure 3. Flattening of a network of geodesic curves following an adaptation of the Compass method, axonometry and top view for case I (tunnel).

holes. Concretely, every node in the flattened grid is doubled, one on each intersecting beam. The two nodes representing a same intersection are allowed to be within a certain distance of each other. At initialisation of the relaxation process, each two nodes are moved by half the allowed distance in opposite directions. At each iteration, they are constrained within a circle of radius equal to the allowed distance and of centre equal to the initially matching beam intersection. This assembly clearance is an added degree of freedom helping the further reduction of embedded elastic energy.

Mesh relaxation can be applied to any grid of beams having anisotropic cross sections. After relaxation, the grid constitutes a flat assembly of beams with minimal bending energy, and whose distances between beam intersections are similar to distances in the target gridshell. However, whether the target gridshell can actually be built from this flat configuration is not checked yet. For instance, for given cross sections and material stiffness, a high curvature along the strong axis might produce stresses exceeding the beam's material limits. The next step addresses such mechanical validation.

\section{Mechanical validation}

In a context of reuse, material properties and component geometries are given from a stock and cannot be prescribed from preliminary structural analyses ${ }^{15,16}$. The objective being the design of a gridshell made of reused skis, the mechanical characteristics of members are those of standard skis. If mechanical requirements are not met, the geometry is not buildable with skis and has to be adapted.

In order to conclude on the ability to assemble the grid in a near-flat configuration, calculations are performed to ensure that stresses in beams are within the elastic domain of skis. Tilting of rectangular beams is accounted for as a mean to reduce the effort required to reach a given curvature. To this end, the effective inertia is computed according to the angle of inclination.

Eventually, mechanical validation on the deformed configuration in space is carried out. Since the resulting gridshell is assumed to be geodesic, members will only be subject to torsion and bending around the weak axis. This guarantees the absence of excessive local stresses and potential geometrical instabilities.

Modelling of the structural behaviour of the gridshell is achieved through Karamba3 $d^{14,17}$. Supports are pinned and connections between members are hinged, assuming a rotation axis oriented normal to the target surface. Considered loads are the self-weight $\mathrm{G}$ - artificially increased by $15 \%$ to consider the weight of the assemblies - and a uniformly distributed snow S. These loads are combined following Eurocode's serviceability and ultimate limit states criteria.

In the next sections, the overall methodology (figure 2) is applied to a series of cases, with target surfaces varying geometries and topologies. Cases are selected to validate the generation process for various degrees and types of curvatures. The three next sections are organised by model type: geometric models for methodological steps from target surface to relaxed flattened grid; mechanical model for the mechanical validation of the transformation; and physical model as a confirmation step.

\section{Geometric models}

\section{Case I (tunnel)}

The first case study is a geodesic gridshell mapped onto a tubular surface with a negative Gaussian curvature (Figure 4.I left). The two-way grid admits only one axial symmetry and is composed of 16 geodesic curves in both directions, with ground supports spaced about $1 \mathrm{~m}$ apart. The height of the gridshell varies from $2.2 \mathrm{~m}$ in the middle to $3.6 \mathrm{~m}$ at the ends. The average and maximum curvatures are $0.210 \mathrm{~m}^{-1}$ and $0.983 \mathrm{~m}^{-1}$ respectively.

The flattened configuration of the geodesic network is computed using method "A" since it allows to be accurate on such curved geometries. Then, mesh relaxation is applied on the flat network of curves to reduce the overall inplane curvature. The relaxation algorithm is computed first with strictly coincident curve node and then with a $20 \mathrm{~mm}$ assembly clearance. The resulting grids from the flattening and the relaxation processes are depicted in Figure 4.I right.

As expected, the introduction of assembly clearance in the model allows to reduce significantly the curvature in the network (Table 1.I). Compared with the other relaxed curve layout without assembly clearance, both average and maximum curvatures are divided by 5 when assembly clearance is taken into account in the model, and by 20 if compared with the initial flattened grid.

\section{Case II (dome)}

The second geodesic gridshell is based on a low-curved dome-like surface with positive Gaussian curvature (Figure 4.II left). The bi-symmetrical dome structure reaches a height of $3.3 \mathrm{~m}$ at its centre and spans $16 \mathrm{~m}$ in the longitudinal direction and $10 \mathrm{~m}$ in the orthogonal one. The network is 

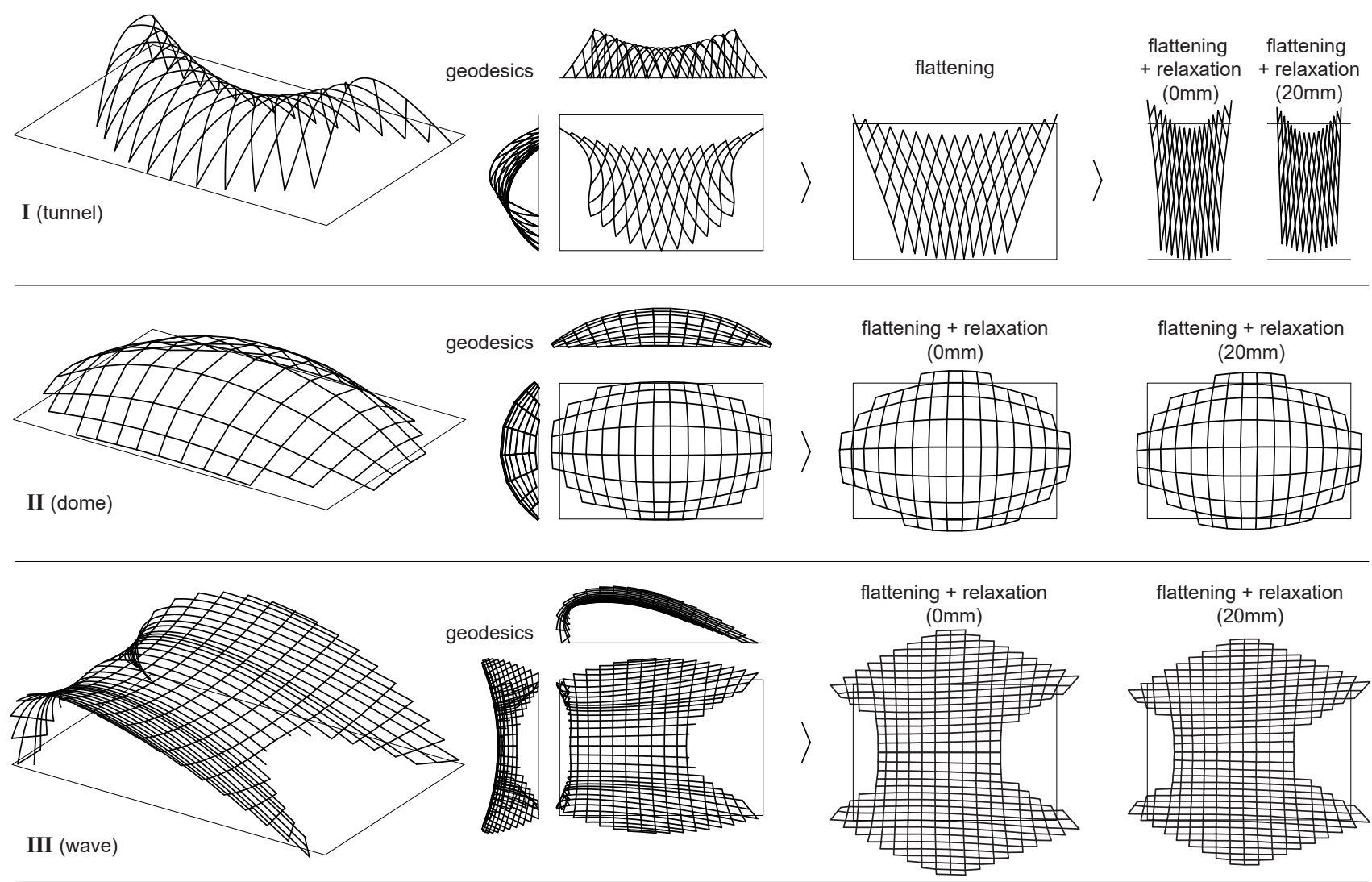

flattening + relaxation
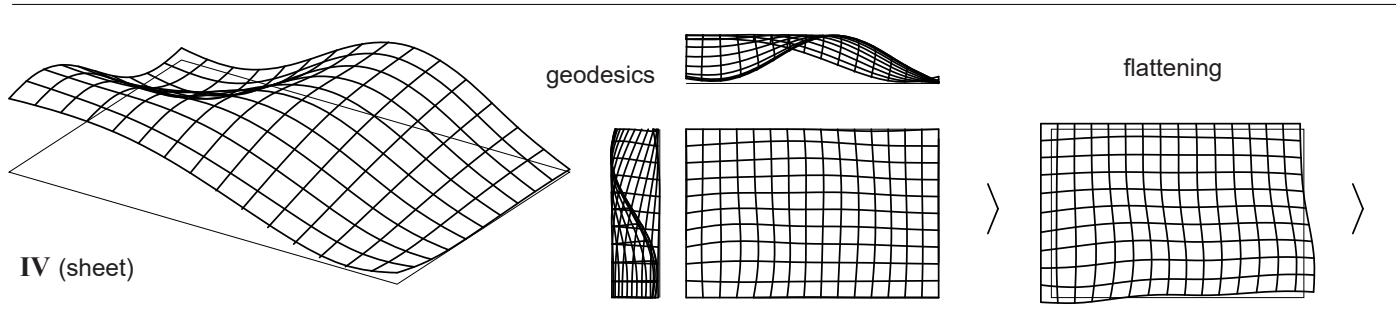

flattening + relaxation
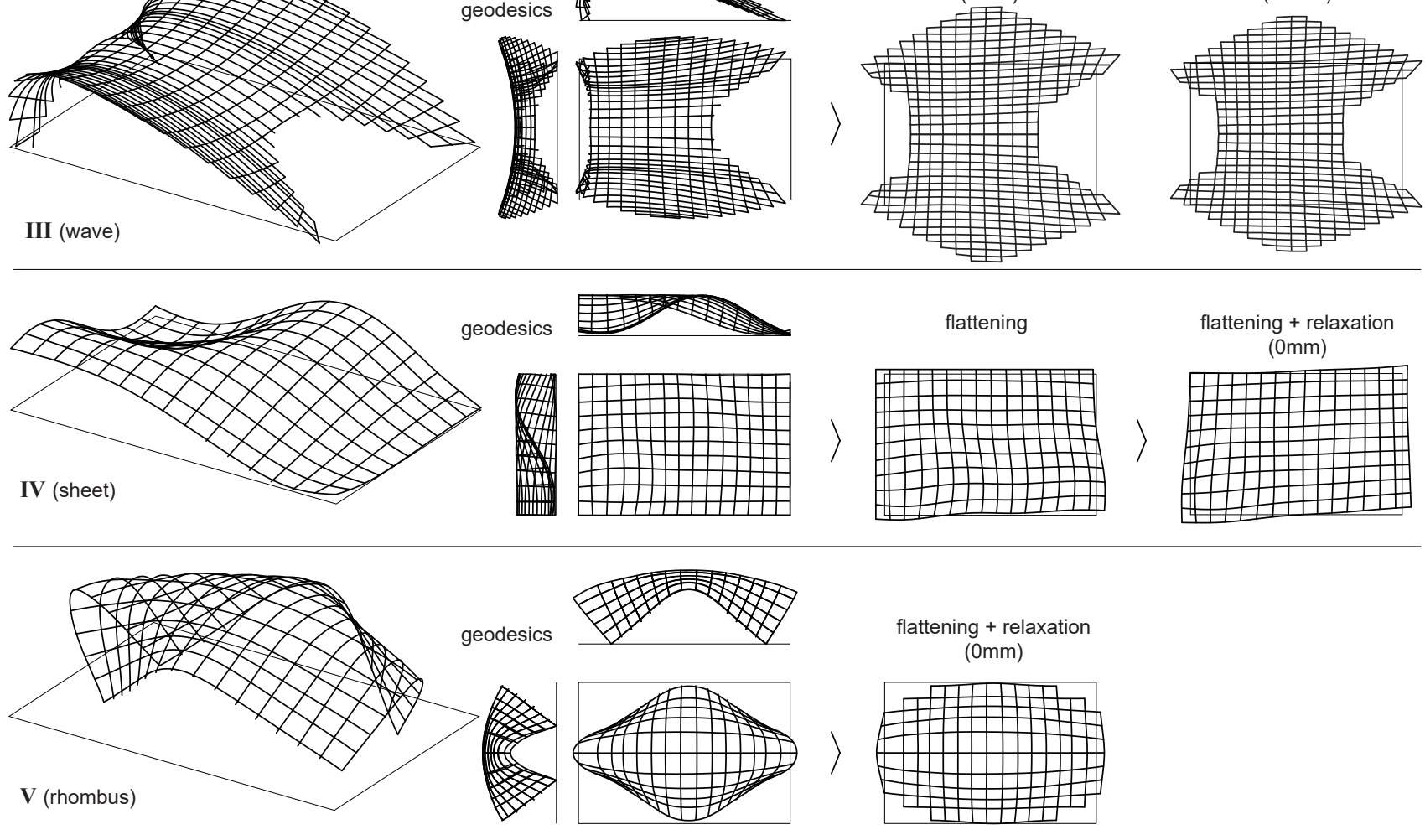

flattening + relaxation
\[ (0 \mathrm{~mm}) \]

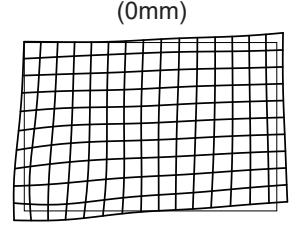

V (rhombus)
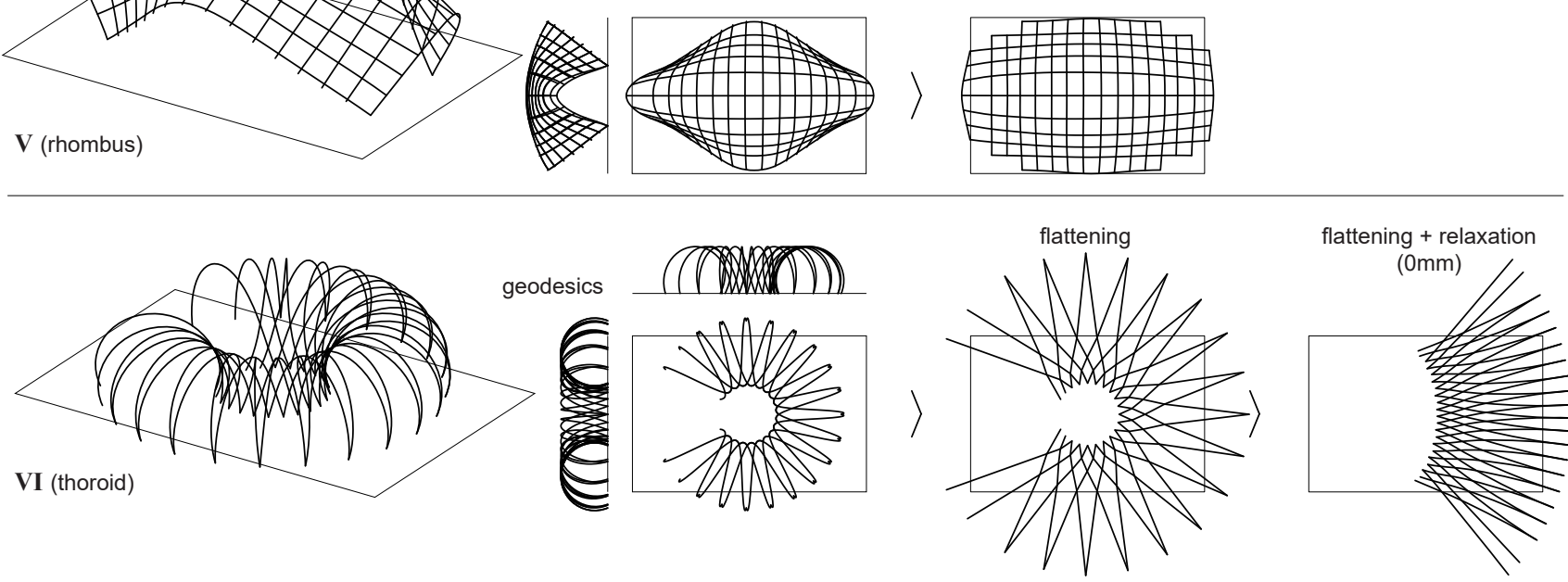

Figure 4. Initial geodesics and results after flattening and/or curvature relaxation for 6 target surfaces. Numbers in brackets corresponds to assembly clearance.

composed of 16 geodesic curves in one direction and 9 in the other. In a similar fashion, the ground support have been designed to be about $1 \mathrm{~m}$ apart. The average and maximum
Gaussian curvatures of the target surface are $0.107 \mathrm{~m}^{-1}$ and $0.321 \mathrm{~m}^{-1}$.

The flattening of the grid was computed using method "B". Since the mesh is symmetrical, regular and lightly 
curved, the creation of the affiliated mesh is simple and accurate. In addition, the mesh flattening algorithm flattens and relaxes the mesh at the same time, thus providing a better initialisation for the relaxation process. Similarly, the mesh relaxation is computed two times, without and with a $20 \mathrm{~mm}$ assembly clearance. Results are depicted in Figure 4.II right. Table 1.II summarises the curvatures obtained with the algorithm.

In this case, the difference of average and maximal curvature between the grids relaxed with and without assembly clearance is not substantial (Table 1.II). This is explained by the double axial symmetry of the shell which constraints the movements of the nodes.

\section{Case III (wave)}

The third case study is a shell based on a surface with negative Gaussian curvature (Figure 4.III left). The geometry is more irregular than the previous two. It is $14.7 \mathrm{~m}$ long, $12.8 \mathrm{~m}$ width, $3 \mathrm{~m}$ high at the centre, $1.6 \mathrm{~m}$ and $2.4 \mathrm{~m}$ high at the lowest openings and 4.2mhigh at the highest openings. The network is composed of 35 geodesic curves in one direction, and 17 in the other. The average and maximum curvatures are $0.111 \mathrm{~m}^{-1}$ and $1.404 \mathrm{~m}^{-1}$.

Method "B" is also applied to flatten this gridshell (Figure 4.III right). In a similar fashion as for case I, the introduction of assembly clearance in the model leads to a reduction of average curvature in the network (Table 1.III).

\section{Case IV (sheet)}

The fourth case is based on a rectangular surface with a transverse crease, similar to a wrinkled sheet (Figure 4.IV left). The sign of the Gaussian curvature changes throughout the surface. The geodesic curves are then drawn with an approximate distance of $1 \mathrm{~m}$ along the surface border. The gridshell is supported by two regions on opposite sides of the crease, and its maximal height is $3 \mathrm{~m}$. The beam network is then flattened and relaxed using method "A" (Figure 4.IV right). Note that on this shape method "B" would also provides satisfactory outputs. Resulting curvatures are given in Table 1.IV.

\section{Case V (rhombus)}

The fifth case maps a surface with a highly changing positive Gaussian curvature (Figure $4 . \mathrm{V}$ left). Towards the corners, the curvature is very high compared with the other parts of the surface. The resulting geodesic gridshell is therefore composed of highly bent members. After flattening of the network using method "B" and mesh relaxation, the maximal curvature remains very high compared to previous case studies (Table 1.V).

\section{Case VI (toroid)}

The sixth case is a $3 \mathrm{~m}$ high toroid geodesic gridshell (Figure 4.VI left). The geodesic lines have been chosen to span from the internal circle to the external one. Consequently, the curve network has a changing density and does not form a bi-directional grid. In this case, method "A" was first applied. The mesh relaxation allowed to significantly reduce the curvature (Table 1.VI), and resulted in a more compact configuration (Figure 4.VI right). case I (tunnel)

\begin{tabular}{|c|c|}
\hline $\begin{array}{c}\text { average } \\
{\left[\mathrm{m}^{-1}\right]}\end{array}$ & $\begin{array}{c}\text { maximum } \\
{\left[\mathrm{m}^{-1}\right]}\end{array}$ \\
\hline
\end{tabular}

\begin{tabular}{|l|c|c|}
\hline initial flat curvature (method A) & 0.021 & 0.061 \\
\hline $\begin{array}{l}\text { relaxed curvature } \\
\text { with no assembly clearance }\end{array}$ & 0.006 & 0.023 \\
\hline $\begin{array}{l}\text { relaxed curvature } \\
\text { with 20mm assembly clearance }\end{array}$ & 0.001 & 0.003 \\
\hline
\end{tabular}

case II (dome)

\begin{tabular}{|l|c|c|}
\hline initial flat curvature (method B) & 0.054 & 0.160 \\
\hline $\begin{array}{l}\text { relaxed curvature } \\
\text { with no assembly clearance }\end{array}$ & 0.045 & 0.112 \\
\hline $\begin{array}{l}\text { relaxed curvature } \\
\text { with 20mm assembly clearance }\end{array}$ & 0.038 & 0.116 \\
\hline
\end{tabular}

\section{case III (wave)}

\begin{tabular}{|l|c|c|}
\hline initial flat curvature (method B) & 0.151 & 1.580 \\
\hline $\begin{array}{l}\text { relaxed curvature } \\
\text { with no assembly clearance }\end{array}$ & 0.110 & 0.798 \\
\hline $\begin{array}{l}\text { relaxed curvature } \\
\text { with 20mm assembly clearance }\end{array}$ & 0.020 & 0.316 \\
\hline
\end{tabular}

case IV (sheet)

\begin{tabular}{|l|l|l|}
\hline initial flat curvature (method A) & 0.03 & 0.13 \\
\hline relaxed curvature & 0.01 & 0.07 \\
\hline
\end{tabular}

\section{case V (rhombus)}

\begin{tabular}{|l|l|l|}
\hline initial flat curvature (method B) & 0.09 & 0.85 \\
\hline relaxed curvature & 0.02 & 0.33 \\
\hline
\end{tabular}

case VI (toroid)

\begin{tabular}{|l|l|l|}
\hline initial flat curvature (method A) & 0.07 & 0.37 \\
\hline relaxed curvature & 0.04 & 0.12 \\
\hline
\end{tabular}

Table 1. average and maximum curvatures before and after relaxation.

\section{Recap}

The flattening of geodesic networks was here made using two different methods: method "A" maintains all initial arc lengths; while method "B" flattens a mesh of quads while allowing slight variations of their edge lengths and angles. The choice between the two is mainly motivated by the shape of the grid. A relaxation method was applied thereafter on the flat grid in order to minimise its elastic bending energy. The applicability of the three methods has been successfully demonstrated on sixth surfaces with varying Gaussian curvatures: negative, positive, or mixed.

\section{Mechanical models}

The previous section applied purely geometric methods in order to flatten a mesh of geodesic curves and relax it to its reduce bending energy. Nonetheless, mechanical calculations are necessary to determine if the maximal curvature achieved in the flat configuration does not exceed the stresses that the material, e.g. reclaimed composite skis, can withhold. Important stresses can either lead to 
plastification if they are superior to the elastic limit, or to buckling if they are superior to the critical limit.

In the following sub-sections, cross-sections of bent skis are first analysed in order to identify critical curvatures and prestress forces to apply. The overall gridshell design, i.e. choice of layer quantities and bracing, is then studied in order to identify maximum vertical displacements.

\section{Critical curvatures of bent skis and assembly prestress}

Material properties According to Truong et al. ${ }^{18}$ and to Rainer et al. ${ }^{19}$, a ski can be treated as a rectangular cross section beam with an average width of $b=75 \mathrm{~mm}$ and an average thickness of $\mathrm{t}=13 \mathrm{~mm}$. Therefore, its strong inertia is $\mathrm{I}_{\mathrm{y}}=45.70 * 10^{-8} \mathrm{~m}^{4}$ and its weak inertia is $\mathrm{I}_{\mathrm{z}}=1.37 *$ $10^{-8} \mathrm{~m}^{4}$ (Figure 6).

A ski Young's modulus is equal to $\mathrm{E}=20 \mathrm{GPa}$, and its torsional stiffness is around GJ $=378 \mathrm{Nm}^{2}$.

Elastic curvature The section modulus of a ski is derived from its inertia: $\mathrm{W}_{\mathrm{el}, \mathrm{y}}=\frac{\mathrm{I}_{\mathrm{y}}}{\mathrm{b} / 2}=12 \cdot 188 \cdot 10^{-6} \mathrm{~m}^{3}$; and $\mathrm{W}_{\mathrm{el}, \mathrm{z}}=$ $\frac{\mathrm{I}_{\mathrm{z}}}{\mathrm{h} / 2}=2 \cdot 113 \cdot 10^{-6} \mathrm{~m}^{3}$.

The yield stress $f_{y}$, defined as the limit of elasticity, corresponds to the yield stress of glass fibre constituent, which is about $0.8 \mathrm{GPa}$. In agreement with the composites design codes, a safety factor of 0.3 is considered, i.e. $\mathrm{f}_{\mathrm{y}}=0.25 \mathrm{GPa}$. Thus, $\mathrm{M}_{\mathrm{el}, \mathrm{y}}=\mathrm{f}_{\mathrm{y}} * \mathrm{~W}_{\mathrm{el}, \mathrm{y}}=3.250 \mathrm{kN} \cdot \mathrm{m}$, and $\mathrm{M}_{\mathrm{el}_{\mathrm{z}}}=0.563 \mathrm{kN} . \mathrm{m}$.

As $\mathrm{K}=\frac{\mathrm{M}}{\mathrm{EI}_{\mathrm{y}}}$, the maximum curvature around the strong axis in elastic behaviour is $\mathrm{K}_{\mathrm{el}, \mathrm{y}}=0.356 \mathrm{~m}^{-1}$. Similarly, $\mathrm{K}_{\mathrm{el}, \mathrm{z}}=$ $2.051 \mathrm{~m}^{-1}$.

Critical curvature The beam lateral torsional buckling is here studied in order to determine whether a ski would be susceptible to instability when assembling the grid flat. The critical moment corresponds to $\mathrm{M}_{\mathrm{cr}, \mathrm{y}}=$ $\frac{\mathrm{C}_{1} \pi}{\mathrm{L}} \sqrt{\mathrm{GJ} * \mathrm{EI}_{\mathrm{Z}}}=910 \mathrm{~N}$.m, with $\mathrm{C}_{1}=1.35$ for a simply supported beam under a point load at midspan, resulting in the following critical curvatures along strong and weak axes: $\mathrm{K}_{\mathrm{cr}, \mathrm{y}}=\frac{\mathrm{C}_{1} \pi}{\mathrm{L}} \frac{\sqrt{\mathrm{GJ}^{2} \mathrm{EI}_{\mathrm{z}}}}{\mathrm{EI}_{\mathrm{y}}} ; \mathrm{K}_{\mathrm{cr}, \mathrm{z}}=\frac{\mathrm{C}_{1} \pi}{\mathrm{L}} \frac{\sqrt{\mathrm{GJ}^{2} \mathrm{EI}_{\mathrm{y}}}}{\mathrm{EI}_{\mathrm{z}}}$.

The buckling length $\mathrm{L}$ corresponds to the interval between two successive geodesics. It is assumed that a geodesic will experience torsional buckling in a similar way as shown for the beam on Figure 5. The first and second geodesics intersecting it act as a support while a force is exerted on the end to assemble it to the third one.

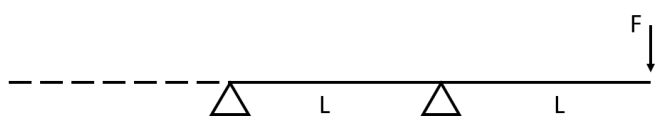

Figure 5. Three-point bending model

The theoretical critical curvature of a ski about its strong and weak axes are presented for study cases I, II, and III in Table 2. These values will be compared to the elastic curvature, and later on, to the design curvature obtained in the parametric models.

\begin{tabular}{|l|c|c|c|}
\cline { 2 - 4 } \multicolumn{1}{c|}{} & $\begin{array}{c}\text { case I } \\
\text { (tunnel) }\end{array}$ & $\begin{array}{c}\text { case II } \\
\text { (dome) }\end{array}$ & $\begin{array}{c}\text { case III } \\
\text { (wave) }\end{array}$ \\
\hline average length L[m] & 1.5 & 1.3 & 0.7 \\
\hline $\mathrm{K}_{\mathrm{cr}, \mathrm{y}}\left[\mathrm{m}^{-1}\right]$ & 0.0996 & 0.1149 & 0.2133 \\
\hline $\mathrm{K}_{\mathrm{cr}, \mathrm{z}}\left[\mathrm{m}^{-1}\right]$ & 19.2 & 22.1 & 41.1 \\
\hline
\end{tabular}

Table 2. Maximum curvature of skis around strong and weak axis

Assembly prestress Since lines on the flat layouts are not perfectly straight, skis will have to bend along their strong axis in order to connect them together during assembly. The prestress force needed to apply such bending moment $\mathrm{F} * \mathrm{~L}$ is equal to $\mathrm{F}=\frac{\mathrm{EI}_{\mathrm{y}]} \mathrm{K}_{\mathrm{y}}}{\mathrm{L}}$. Table 3 shows the resulting stresses that would have to be enforced to assemble the planar ski grid in practice.

\begin{tabular}{|l|c|c|c|}
\cline { 2 - 4 } \multicolumn{1}{c|}{} & $\begin{array}{c}\text { case I } \\
\text { (tunnel) }\end{array}$ & $\begin{array}{c}\text { case II } \\
\text { (dome) }\end{array}$ & $\begin{array}{c}\text { case III } \\
\text { (wave) }\end{array}$ \\
\hline average force [N] & 6 & 141 & 261 \\
\hline maximum force [N] & 18 & 816 & 4126 \\
\hline
\end{tabular}

Table 3. Average and maximum forces that must be applied in order to bend skis and assemble them flat.

These results are only valid for flat skis, i.e. when only its strong inertia governs its horizontal curvature. When the ski is initially tilted, the vertical inertia becomes a function of both the strong and weak inertia and the latter decreases with the tilt angle: $\mathrm{I}_{\mathrm{Gz}}=\cos ^{2}(\vartheta) \mathrm{I}_{\mathrm{Gy}}+\sin ^{2}(\vartheta) \mathrm{I}_{\mathrm{Gz}}-$ $2 \cos (\vartheta) \sin (\vartheta) I_{\mathrm{Gyz}}$ As shown on figure 6, the vertical inertia increases up to $30^{\circ}$ of tilt and then decreases. A reduction of vertical inertia will be achieved when considering large angles, which makes the assembly more difficult.

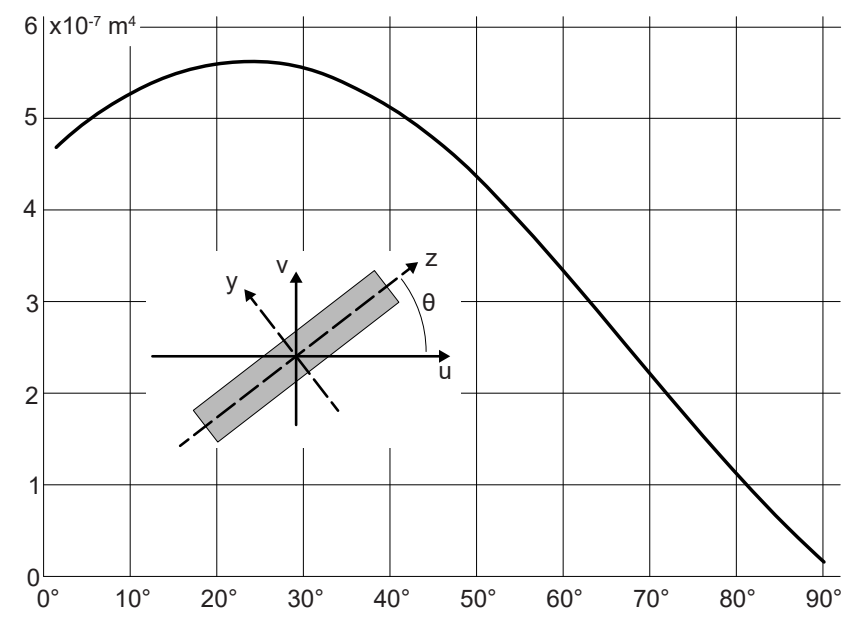

Figure 6. Strong inertia for varying angles of tilting.

In conclusion, it can be assumed in this study that the skis remain flat on the ground during assembly and that the prestress needed to connect skis together can be exerted manually by hand. Moreover, calculations show that, on the minor axis, $\mathrm{K}_{\mathrm{el}, \mathrm{z}}<\mathrm{K}_{\mathrm{cr}, \mathrm{z}}$, meaning that the ski would exceed its elastic limit and plasticises before it would buckle. This must be accounted for in the deformed configuration where minor axis bending is way higher than in the flat configuration. It can also be noticed that, on the strong 
axis, $\mathrm{K}_{\mathrm{cr}, \mathrm{y}}<\mathrm{K}_{\mathrm{el}, \mathrm{y}}$, buckling occurs before yield, which is susceptible to happen in the flat configuration only, as the deformed configuration avoids major axis bending.

\section{Number of layers, bracing, and stiffness}

The following design choices remain available when considering the full gridshell and its mechanical behaviour: the number of layers of skis; the orientation and density of bracing skis. These choices impact the overall stiffness of the structure, and hence vertical displacements under selfweight and applied loads. The following two sections first describe design choices and assumptions. They then analyse the resulting gridshell and discuss their deflection.

Model assumptions While the geometric model started from a target surface to identify the best grid flat on the ground, the erection process follows the opposite sequence: lines of skis are first assembled flat on the ground and then erected to form the target surface. The bracing, also made with skis, would be assembled to the geodesic grid structure thereafter. The mechanical model accounts for this third network.

Supports are modelled as pinned to the ground and connections as hinges whose axis of rotation are the normal vectors of the underlying surface, or equivalently, the normal vector of the intersecting curves. As they are not following geodesic curves, bracing skis would not be connected to each other. There would be one ski for each diagonal, so that it remains flat, without undesired bending moment on the strong axis.

As presented before, material properties considered for the skis are:

$$
\begin{aligned}
& \text { - } \mathrm{E}=20 \mathrm{GPa} . \\
& \text { - } \mathrm{G}=6.89 \mathrm{GPa} \\
& \text { - } \rho=13.4 \mathrm{~kg} / \mathrm{m}^{3} \text {. } \\
& \text { - } \mathrm{f}_{\mathrm{y}}=0.25 \mathrm{GPa} \text {. }
\end{aligned}
$$

Two loads are considered for the design. The first one is the self weight $\mathrm{G}$ enhanced by $15 \%$ to consider the weight of connections. The second one is snow $\mathrm{S}=0,4 \mathrm{kN} / \mathrm{m}^{2}$. Eurocode combinations are used to combine these loads for both serviceability $(\mathrm{G}+\mathrm{S})$ and ultimate $(1.35 \mathrm{G}+1.5 \mathrm{~S})$ limit states.

The following sub-sections computes deflections for the first three case studies and various options of bracing and numbers of layers. Considering that a ski is around $1.5 \mathrm{~m}$ long it is possible to get an order of magnitude on the number of skis required.

case I (tunnel) Figure 7 illustrates the pre-design models of the first structure (a) without bracing, (b) with bracing on the short diagonal and (c) with bracing on the long diagonal. In practice, the latter solution may not mechanically behave as a gridshell but as an arch braced by geodesics, so loads are applied on the arches instead of the geodesic curves. As summarised on Table 4, deflections are computed for one or two layers of skis on the geodesic curves, and for zero or one layer of bracing skis. Results on Table 4 highlight

\begin{tabular}{|c|c|c|c|}
\hline & $\begin{array}{l}\text { deflection } \\
{[\mathrm{mm}]}\end{array}$ & $\begin{array}{c}\text { utilisation } \\
\text { [\%] }\end{array}$ & $\begin{array}{c}\text { \# of skis } \\
{[-]}\end{array}$ \\
\hline \multicolumn{4}{|l|}{ case I (tunnel) } \\
\hline $\begin{array}{l}\text { single layer } \\
\text { no bracing }\end{array}$ & 405 & 25 & 168 \\
\hline $\begin{array}{l}\text { single layer } \\
\text { bracing on short diag. }\end{array}$ & 85 & 14 & 228 \\
\hline $\begin{array}{l}\text { single layer } \\
\text { bracing on long diag. }\end{array}$ & 329 & 25 & 245 \\
\hline $\begin{array}{l}\text { double layer } \\
\text { no bracing }\end{array}$ & 17 & 7 & 336 \\
\hline $\begin{array}{l}\text { double layer } \\
\text { bracing on short diag. }\end{array}$ & 8 & 4 & 396 \\
\hline $\begin{array}{l}\text { double layer } \\
\text { bracing on long diag. }\end{array}$ & 229 & 21 & 322 \\
\hline
\end{tabular}
the influence of bracing on the structure stability as it fixes the form. Bracing on the short diagonal reduces overall deflection by a factor close to six and reduces utilisation by case II (dome)

\begin{tabular}{|l|c|c|c|}
\hline $\begin{array}{l}\text { single layer } \\
\text { no bracing }\end{array}$ & 202 & 48 & 176 \\
\hline $\begin{array}{l}\text { single layer } \\
\text { bracing }\end{array}$ & 50 & 23 & 297 \\
\hline $\begin{array}{l}\text { double layer } \\
\text { no Bracing }\end{array}$ & 82 & 27 & 352 \\
\hline $\begin{array}{l}\text { double layer } \\
\text { bracing }\end{array}$ & 47 & 24 & 473 \\
\hline
\end{tabular}

\section{case III (wave)}

\begin{tabular}{|l|c|c|c|}
\hline $\begin{array}{l}\text { single layer } \\
\text { no bracing }\end{array}$ & 60747 & 4963 & 376 \\
\hline $\begin{array}{l}\text { single layer } \\
\text { bracing }\end{array}$ & 400 & 106 & 910 \\
\hline $\begin{array}{l}\text { double layer } \\
\text { no bracing }\end{array}$ & 12345 & 1302 & 752 \\
\hline $\begin{array}{l}\text { double layer } \\
\text { bracing }\end{array}$ & 221 & 60 & 1286 \\
\hline
\end{tabular}

Table 4. Displacement, utilisation and number of skis for each gridshell typology.

a factor of two. Also, adding a second layer to the structure makes the structure stiffer and reduces the stresses in the skis.

The serviceability limit state (SLS) criterion for displacements is L/300 where the length of the geodesic beams is around $10 \mathrm{~m}$, meaning that deflection must not exceed $33 \mathrm{~mm}$. Only the double-layered grid without bracing and with bracing on the short diagonal respect this criterion. Every configuration respect the ultimate limit state (ULS) criterion. Thus, two layers of skis with bracing on the short diagonal ensures the Tunnel gridshell feasibility.

case II (dome) Figure 8 shows the pre-design models of the second structure without and with bracing. In this case, bracing along one diagonal or the other has an identical effect on the overall behaviour, since the grid is symmetrical.

The influence of bracing on the displacement is also striking in this case: the deflection is divided by seven when the structure is braced. Geodesic beams with the highest deflection are $17 \mathrm{~m}$ long, the limit is $56 \mathrm{~mm}$. A single layer of skis with bracing is therefore sufficient to meet both SLS and ULS criteria, hence ensuring its feasibility. 


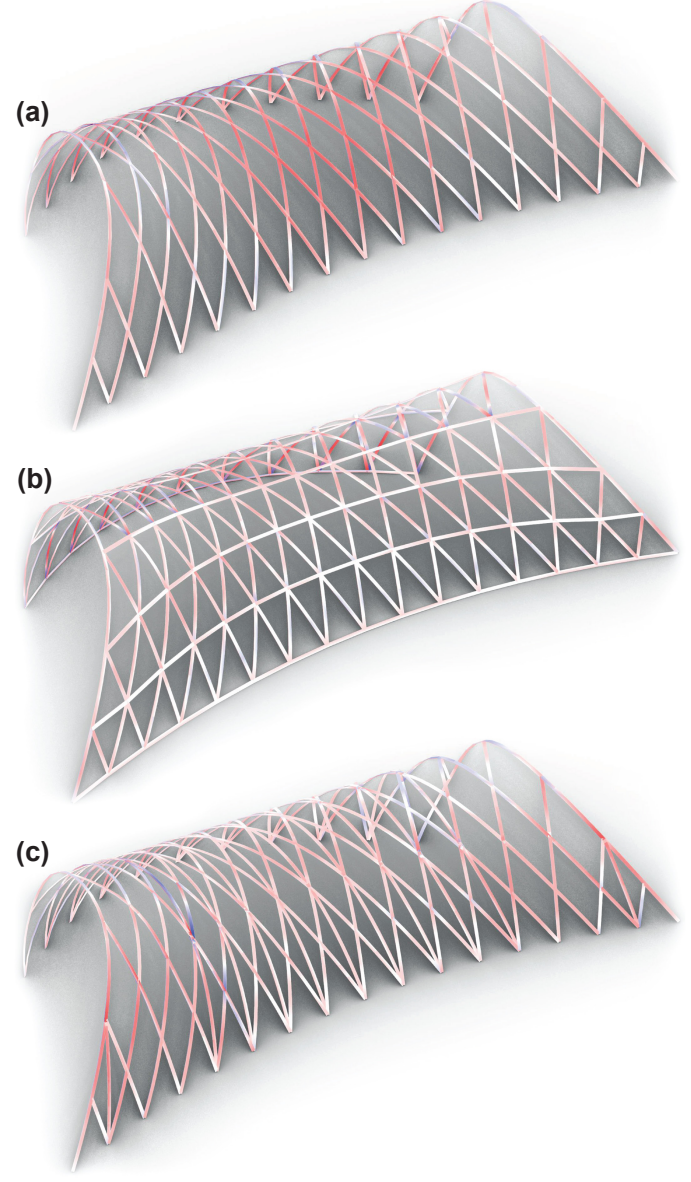

tension

$24.3 \%$

$18.3 \%$

$12.2 \%$

$\square 6.1 \%$

$\begin{array}{r}\square .9 \% \\ \hline\end{array}$

$11.8 \%$

$17.7 \%$

compression

tension

$\longrightarrow 13.8 \%$

10.3\%

$6.9 \%$

$3.4 \%$

$3.4 \%$
$3.1 \%$

$6.3 \%$

$6.3 \%$
$9.4 \%$

$12.5 \%$

compression

tension

$25.0 \%$

$18.7 \%$

$12.5 \%$

$6.2 \%$

$0.0 \%$

$11.7 \%$

$17.5 \%$

$23.3 \%$

Figure 7. Bracing layouts and material utilisation in case I: (a) without bracing; (b) with bracing on short diagonals; (c) with bracing on long diagonals.
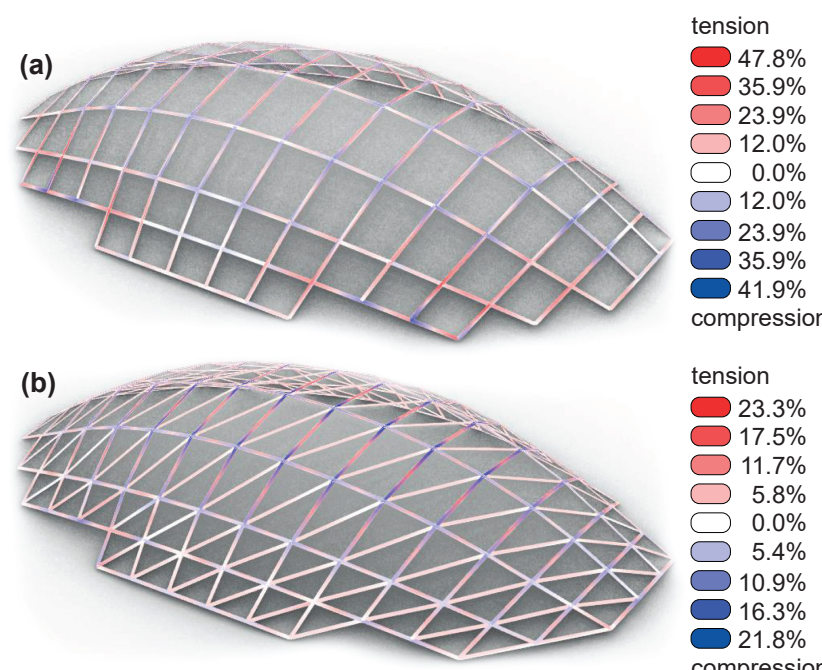

Figure 8. Bracing layouts and material utilisation in case II: (a) without bracing; (b) with bracing.

case III (wave) Figure 9 shows the pre-design models of the third gridshell with and without bracing. Similarly, symmetry allows to study only one bracing solution.

The length considered for the deflection is $13 \mathrm{~m}$, which sets the SLS limit to $43 \mathrm{~mm}$. None of the prototypes respect this criterion. A solution would be to add more supports, it would also reduce the utilisation since the pre-design model shows that it is maximum in the area close the supports. Another solution would be to increase the curvature in the long direction to make the structure behave like an arch.
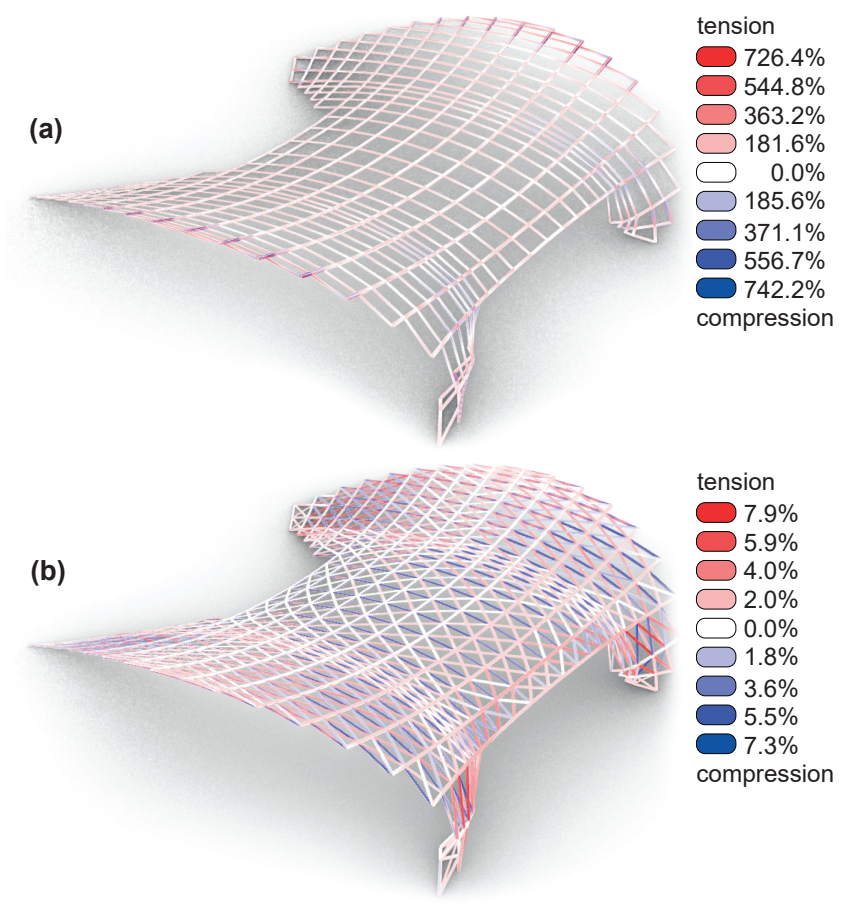

Figure 9. Bracing layouts and material utilisation in case III: (a) without bracing; (b) with bracing.

\section{Physical model}

Reduced-scaled physical models here aim at highlighting the possibility to assemble geodesic curves fully on the ground. Organic glass rods with a rectangular cross-section are used to approximate the behaviour of skis. They are drilled and connected together with upholstery nails and earring clasps to ensure tangency between intersecting beams. The scale is $1: 14$ making the longest rods in the model $70 \mathrm{~cm}$ long.

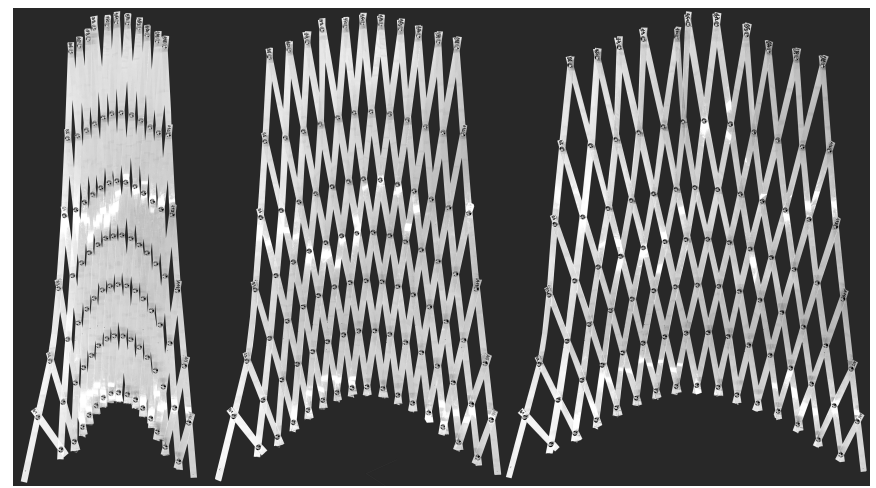

Figure 10. Case I (tunnel) model deployment on the ground.

Figure 10 highlights that the rods rather bend out of the plane than bend around their strong axis, this was not shown on the simulations but was expected. It is well visible for the two exterior rods and some rods in the middle of the network on the right picture.

Bending skis on their strong axis during flat-assembly remains feasible as long as the deflection does not exceed 

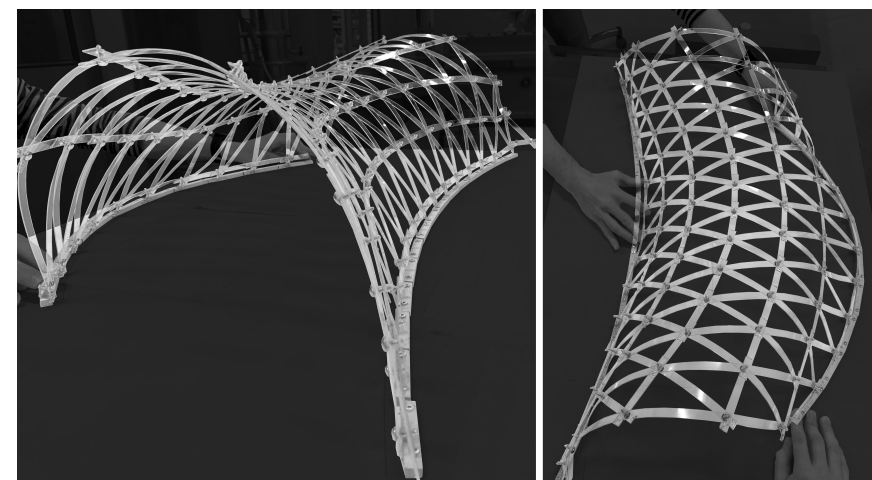

Figure 11. Case I (tunnel) model deployment off the ground connection to the supports to obtain the final shape.

a few tens of centimetres. In practice, at low height, it is still possible for a person to assemble the structure in a nearly, but not fully, flat configuration without using scaffolding. Figure 11 emphasises the importance to fix the grid strongly to the supports to give its final shape. Indeed, this does not correspond to a minimum of energy so there must be foundations to lock the form.

\section{Discussion}

\section{Comparison of the parametric model and mechanical calculation results}

Results show that, for a regular ski section, the elastic moment is lower than the critical moment, meaning that the ski breaks - or even breaks - before buckling.

When deployed, the maximum curvature of the geodesics on their weak axis is $0.983 \mathrm{~m}^{-1}$ for case I (tunnel), $0.321 \mathrm{~m}^{-1}$ for case II (dome), and $1.404 \mathrm{~m}^{-1}$ for case III (wave). All these curvatures are inferior to $2.051 \mathrm{~m}^{-1}$, the elastic limit, so the shape created can be approximated by bending skis on their weak axis.

Furthermore, when assembled flat, the maximum curvature on their strong axis is $0.003 \mathrm{~m}^{-1}$ for case I (tunnel), $0.116 \mathrm{~m}^{-1}$ for case II (dome), and $0.316 \mathrm{~m}^{-1}$ for case III (wave). They are inferior to $0.356 \mathrm{~m}^{-1}$. Hence, it is possible to assemble these gridshells from planarised geodesic curves.

\section{Limits of the flattening methods}

Using Method "A", the construction of the grid can reveal difficult, especially because two circles can have two intersection points and it is thus necessary to automatically select the correct one between these two. To prevent any error, hand adjustments can be provided. Nonetheless, as shown previously, it has been tested on various examples, showing that it is flexible and can be adapted to many configurations.

Method "B" is efficient as it flattens and relaxes the grid at the same time, but it can be difficult to implement if geodesic polylines have a different number of segments. The situation occurred in case III (wave) for which a Python ${ }^{20}$ script was added to the parametric modelling process in order to deal with the number of segments, varying between a geodesic and another. Figure 12 exhibits the issue of polylines with varying number of segments. The difficulty is to define a proper mesh from polylines that does not always have the same length. The solution displayed is the one used to mesh the case III (wave), the first nodes of longer geodesic are not taken into account to define quadrangular faces.

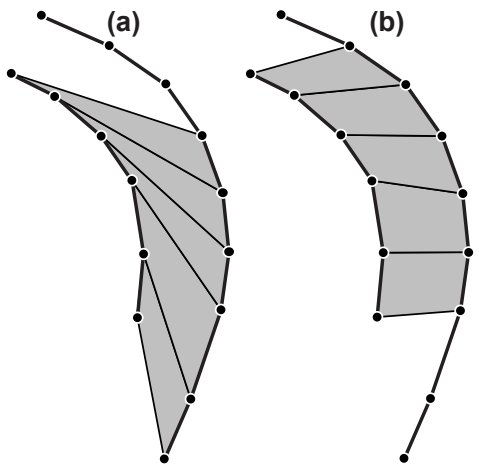

Figure 12. Mesh flattening issue when varying number of segments between polylines: a) Quadrangular faces not relevant b) Quadrangular faces redefined

Mesh relaxation only minimises bending energy in the plane, it does not allow the grid to go off the ground. In practice, it is acceptable to assemble a non-planar grid as long as the beams height does not require scaffolding. The physical models highlight that some organic glass beams prefer bending off the ground in their weak axis than bending on their strong axis. This suggests, and confirms the intuition that giving a certain out-of-plane displacement capacity in the flat configuration can lead to a grid with a lower bending energy.

\section{Limits of the numerical simulations}

The presented design approach is not exhaustive. In practice the structure is bending active with prestress due to bending, so in those results displacements may be overrated, and the utilisation may be underrated.

Moreover, only self-weight and snow loads have been considered. Up-draft, down-draft, and lateral wind must be added for further design. Ski is considered straight and its cross-section geometries and material properties constant which is not the case for most skis.

\section{Limits of the physical models}

The material used for physical models (Figure 11) is different from the wood-and-glass fibre composite material of a ski. Moreover, the uncertainty in the drilling of the rods may lead to an error in the position of the intersections. Therefore, this model aims at proving the flat-assembly process more than checking the structural behaviour of the gridshell. The model also allows to better understand how the flatten geodesics behave in practice compared to the initial parametric modelling.

As far as case I (tunnel) is concerned, the physical model confirms the possibility to assemble a flat grid and deploy it, which supports the reliability of the methods presented.

\section{Conclusion}

Elastic gridshells designed with geodesics are very attractive as they can be built with either isotropic or anisotropic beams tangent to the target surface. In this context, skis, which 
require strength and high flexibility for their primary use as sport equipment, are interesting building materials that can be reclaimed.

The main purpose of this study is to highlight the feasibility of assembling a gridshell flat by developing two parametric modelling methods.

The first method, method "A", is based upon the compass method commonly used to draw Chebychev networks on surfaces. It has been here extended to map networks whose segments do not have the same length. The resulting flat network does not however minimise the curvature of the elements. Thus, a mesh relaxation process is used afterwards to obtain the minimal bending energy configuration. Results on three different gridshells highlight that the curvature can be divided by a up-to- 20 factor.

The second method, method "B", defines a mesh from geodesic polylines and planarises it while ensuring soft constraints on lengths and angles. With this method, the resulting flat grid already presents minimal bending energy.

Six case studies with varying geometries and topologies are chosen to test this building process efficiency. Mechanical calculations on the skis showed that, on the weak axis, yield occurs prior to buckling, which is more susceptible to happen in the deformed configuration than in the flat configuration. On the strong axis, buckling occurs prior to yield, but this can only happen in the flat configuration where major axis bending is induced. Thus, since this elastic limit is not reached during the flatassembling process of neither of the three first gridshells, it is concluded that they can be built from a flat configuration and the erected into their target surface. Design options, i.e. choice of number of layers and bracing layout, were also evaluated in order to assess the structures' satisfaction of serviceability and ultimate limit state criteria.

A physical model was used to validate the flattening methods in real settings. It confirmed that the nearly-flat assembly and deployment of a grid made of anisotropic beams is possible, at least for case I (tunnel). The most striking difference is the tendency for off-plane bending of the rods rather than for bending around their strong axis, which confirms the relevance of the two flattening methods. Studying off-plane bending further would allow to determine the conditions allowing the grid to be assembled nearly-flat on the ground. The idea is that if the bars bend below a certain height, the assembly would still be possible without scaffolding.

\section{References}

1. Liddell I. Frei otto and the development of gridshells. Case Studies in Structural Engineering 2015; 4: 39-49. DOI: 10.1016/j.csse.2015.08.001.

2. Harris R, Romer J, Kelly $\mathrm{O}$ et al. Design and construction of the downland gridshell. Building Research \& Information 2003; 31(6): 427-454. DOI:10.1080/0961321032000088007.

3. Baverel O, Caron JF, Tayeb F et al. Gridshells in composite materials: Construction of a $300 \mathrm{~m}(2)$ forum for the solidays' festival in paris. Structural Engineering International 2012; 22: 408-414. DOI:10.2749/101686612X13363869853572.
4. Colabella S, D'Amico B, Hoxha E et al. Structural design with reclaimed materials: An elastic gridshell out of skis. In IASS 2017: Interfaces: Architecture, Engineering, Science. pp. 1-10.

5. Bouhaya L, Baverel O and Caron JF. Optimization of gridshell bar orientation using a simplified genetic approach. Structural and Multidisciplinary Optimization 2014; 50(5): 839-848. DOI:10.1007/s00158-014-1088-9.

6. Maia AR, Baverel O and Lebée A. Design strategies for gridshells with singularities. Journal of the International Association for Shell and Spatial Structures 2019; 60(3): 189-200. DOI:10.20898/j.iass.2019.201.023.

7. Douthe C, Mesnil R, Orts $\mathrm{H}$ et al. Isoradial meshes: Covering elastic gridshells with planar facets. Automation in Construction 2017; 83: 222-236. DOI:10.1016/j.autcon.2017.08.015.

8. Pirazzi $\mathrm{C}$ and Weinand $\mathrm{Y}$. Geodesic lines on free-form surfaces - optimized grids for timber rib shells. In World Conference in Timber Engineering WCTE, Portland, USA, 2006. p. 595-601.

9. Soriano E, Sastre R and Boixader D. G-shells: Flat collapsible geodesic mechanisms for gridshells. In IASS 2019: Form and Force. p. 1894-1901.

10. Lagally M. Über die Verbiegung geodätischer Netze, Sitzungsberichte, volume 10. München: Verlag der Königlich Bayerischen Akademie der Wissenschaften, 1910.

11. Pillwein S, Leimer K, Birsak M et al. On elastic geodesic grids and their planar to spatial deployment. ACM Transactions on Graphics 2020; 39: 125. DOI:10.1145/3386569.3392490.

12. Otto F, Hennicke J and Matsushita K. Gitterschalen Gridshells, $I L$, volume 10. Stuttgart: Institut für Leichte Flächentragwerke, 1974.

13. Piker D. Kangaroo physics, 2011. URL https: //www. food4rhino.com/app/kangaroo-physics. Accessed: 2020-12-01.

14. McNeel R and Associates. Rhinoceros, 1992. URL https: //www.rhino3d. com. Accessed: 2020-12-01.

15. Brütting J, Desruelle J, Senatore $G$ et al. Design of truss structures through reuse. Structures 2019; 18: 128-137. DOI: 10.1016/j.istruc.2018.11.006.

16. Fivet $\mathrm{C}$ and Brütting J. Nothing is lost, nothing is created, everything is reused: structural design for a circular economy. The Structural Engineer 2020; 98(1): 74-81.

17. Preisinger C. Karamba 3d. URL https://www. karamba3d. com. Accessed: 2020-12-01.

18. Truong J, Brousseau $\mathrm{C}$ and Desbiens A. A method for measuring the bending and torsional stiffness distributions of alpine skis. Procedia Engineering 2016; 147: 394-400. DOI: 10.1016/j.proeng.2016.06.326.

19. Rainer F, Nachbauer W, Schindelwig K et al. On the measurement of the stiffness of skis. In Science and Skiing III. pp. 136-147.

20. van Rossum Guido. Python, 1991. URL https://www . python. org. Accessed: 2020-12-01. 